\title{
Role of Immunotherapy in the Management of Locally Advanced and Recurrent/Metastatic Cervical Cancer
}

\author{
Brandon A. Dyer, MDa; Dmitriy Zamarin, MD, PhD ${ }^{b}$; Ramez N. Eskandar, MD; and Jyoti M. Mayadev, MD
}

\begin{abstract}
Despite combined therapeutic approaches, there is an unmet clinical need to identify effective strategies for improved patient outcomes in treating locally advanced and metastatic cervical cancer (CC). Immunotherapy is emerging as a novel therapeutic approach in this disease for which the causative agent, human papillomavirus (HPV), has dynamic, complex immunomodulatory effects. This review explores the biologic rational of immuno-oncology in the treatment of $\mathrm{CC}$ and discusses the initial clinical efficacy, ongoing clinical trials, and rationale for combined multimodal treatment approaches for locally advanced and recurrent/metastatic CC. The utility of immune checkpoint inhibitors is explored, including anti-cytotoxic T-lymphocyte antigen-4 (CTLA-4), PD-1, and PD-L1. Preliminary data supporting the combination of radiotherapy and immunotherapy and areas of active drug development for CC are also reviewed.
\end{abstract}

J Natl Compr Canc Netw 2019;17(1):91-97 doi: $10.6004 /$ jnccn.2018.7108

aDepartment of Radiation Oncology, University of California Davis, Sacramento, California; ${ }^{b}$ Department of Medicine, Memorial Sloan Kettering Cancer Center, New York, New York; and 'Division of Gynecologic Oncology، Department of Obstetrics, Gynecology, and Reproductive Services, and dDepartment of Radiation Medicine, University of California San Diego, La Jolla, California.

\section{Clinical Rationale to Explore Immunotherapy in Cervical Cancer}

Cervical cancer (CC) affects an estimated 12,900 women and accounts for 4,100 deaths annually in the United States, and is the most common gynecologic cancer worldwide. ${ }^{1}$ The addition of cytotoxic systemic chemotherapy to radiation therapy (CRT) for the treatment of CC resulted in a significant improvement in disease-free and overall survival $(\mathrm{OS}) .^{2-7}$ However, patients with stage III/IV disease have a poor prognosis despite CRT, with 4-year progression-free survival (PFS) and OS rates of $51 \%$ and $55 \%$, respectively. ${ }^{5}$ Furthermore, patients with para-aortic lymph node metastases continue to have poor prognoses, with 3 -year PFS and OS rates of $34 \%$ and $39 \%$, respectively. ${ }^{8,9}$ Metastatic and recurrent CC has a median survival of 17 months with standard-of-care frontline platinum/taxane-based chemotherapy and bevacizumab. ${ }^{10}$ Therefore, for most node-positive locally advanced and metastatic CC, an unmet therapeutic need exists. Immuno-oncology has emerged as a potential novel strategy to improve outcomes in patients with CC. Strategies inclusive of adoptive T-cell therapy and immune checkpoint inhibition (ICI) have shown promising objective response rates and durable survival in patients whose disease fails to respond to standard therapy. ${ }^{11,12}$ This review highlights the role of immunotherapy in the management of CC, including the immunology of human papillomavirus (HPV) infection and rationale for immunomodulatory therapeutic approaches, such as the role of tumor-infiltrating lymphocytes (TILs) and the immune checkpoint receptors cytotoxic T-lymphocyte antigen-4 (CTLA4), PD-1, and PD-L1. The immunomodulatory effects of radiation therapy (RT) are also reviewed. Furthermore, clinical data on actionable immunologic targets, such as therapy with anti-CTLA-4, anti-PD-1, anti-PD-L1, vaccine therapy, and adoptive T-cell therapy, are discussed.

See JNCCN.org for supplemental online content. 


\section{Key Elements of Immunomodulation}

Immunology of HPV Infection and T-Cell Detection $\mathrm{CC}$ has been identified as a direct consequence of infection by specific oncogenic HPV viral subtypes, such as 16 and 18..$^{13-15}$ Most individuals clear the infection, but in persistence, the primary viral oncoproteins E6 and E7 are responsible for inactivation of p53 and pRB. ${ }^{16}$ These oncoproteins are thought to modify the cell cycle, resulting in cellular immortalization and clinically apparent CC. ${ }^{16}$

$\mathrm{T}$ cells play a central role in the control of viral infections and prevention of virus-associated tumors. The immune response to tumor and viral antigens is dependent on dendritic cell presentation of antigen peptides to antigen-specific $\mathrm{T}$ cells. These steps are regulated by a complex series of activating and inhibitory signals (Figure 1) ${ }^{17}$ $\mathrm{T}$ cells recognize infected cells and are classically activated via 3 well-defined signals: (1) interaction of T-cell receptors (TCRs) and peptide epitopes of tumor-associated

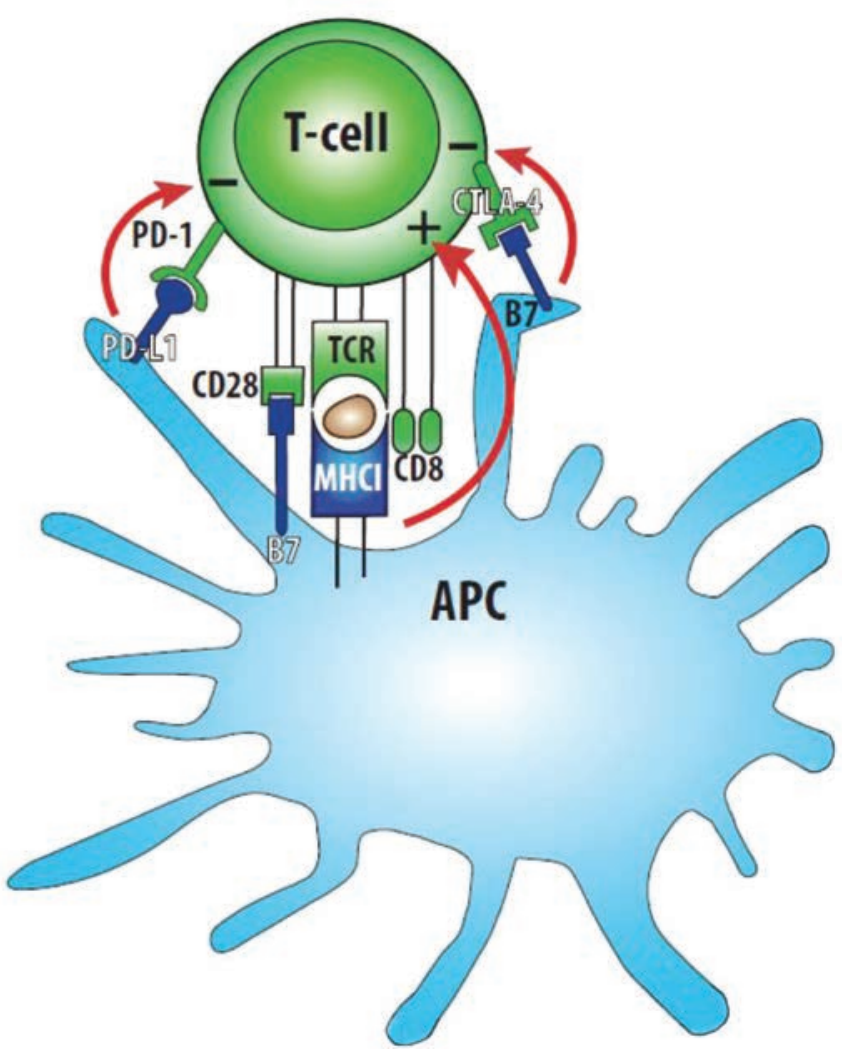

Figure 1. Schematic representation of activating and inhibitory signals between a T cell and an antigen-presenting cell (APC). T-cell activation requires multiple ligand:receptor interactions, including the APC major histocompatibility complexes (MHCl):T-cell receptor (TCR), and B7.1, 2 (ie, CD80/CD86):CD28. T-cell activation can be inhibited by other receptor:ligand interactions (PD-1:PD-L1 and/ or B7:cytotoxic T-lymphocyte antigen-4 [CTLA-4]). These receptor:ligand targets are potentially important therapeutic targets for immunotherapy. antigens and tumor-specific antigens bound to major histocompatibility complexes ${ }^{18}$ of antigen-presenting cells (APCs; dendritic cells, macrophages, Langerhans cells, and B cells); (2) interaction between costimulatory ligands on APCs and their cognate receptors on T cells; and (3) inflammatory cytokine signals. T-cell involvement in HPV infection has been supported by studies showing spontaneous regression of HPV-induced papillomas and increased HPV-related malignancies in immunosuppressed and immunodeficient patients..$^{19,20}$ The development of invasive cancer in the setting of HPV infection is dependent on acquisition of immunosuppressive mechanisms (or immune exhaustion/anergy) in the tumor microenvironment, which drive tumor immune evasion (Figure 2).

\section{Mechanisms of Immune Inhibition}

\section{CTLA-4}

CTLA-4 is a receptor expressed by activated T lymphocytes that counteracts the costimulatory pathway triggered by CD28 ${ }^{21}$ (Figure 1). Although CTLA-4 and CD28 share the same ligand (CD80/CD86), CTLA-4 has significantly greater binding affinity and preferentially binds to CD80/CD86, resulting in deactivation of the immune response. ${ }^{22}$ Therapeutically blocking this mechanism has proven to be an effective clinical strategy in several malignancies. ${ }^{23}$ CTLA-4 blockade may exert its antitumor response via enhanced T-cell-mediated cytotoxicity due to immune deregulation and enhanced T-cell proliferation, and may have direct inhibitory effects on immunosuppressive FOXP3+ regulatory T-cells (Tregs). ${ }^{24}$

\section{$P D-1$}

PD-1 (D279), a key component of the immune regulatory system, is expressed on activated T cells and is a member of the CD28 family of T-cell costimulatory receptors. When PD-1 is bound to its ligands, PD-L1 and PD-L2, which can be expressed on tumor cells and APCs in the tumor microenvironment, T-cell activation is inhibited, progressing to exhaustion, dysfunction, and apoptosis.

Although the CTLA-4:CD80/86 (B7) pathway appears critical for immune priming and acts to attenuate activation signals to regulate the magnitude of early activation of naïve and memory T cells, the PD-1/PD-L1 pathway appears dominant in the effector phase of the immune response, as shown in Figure 1..$^{25-27}$

\section{PD-L1}

PD-L1 has been identified to be overexpressed in as many as $20 \%$ to $50 \%$ of human malignancies, presenting itself as a potential therapeutic target. ${ }^{28} \mathrm{PD}-\mathrm{Ll}$ can be found on both tumor cells and tumor-infiltrating immune cells, with expression levels being higher on immune cells, and levels have been correlated with poor clinical prog- 


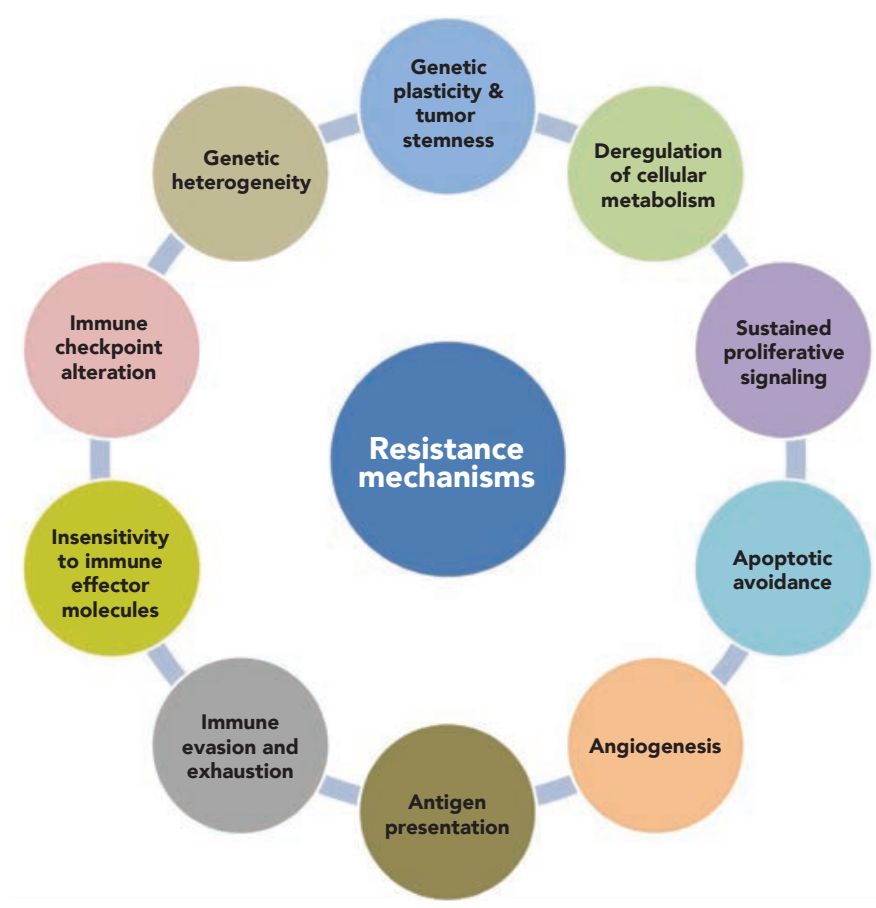

Figure 2. Mechanisms of de novo and acquired immunoresistant niches.

nosis. ${ }^{29-33}$ Inhibitors of PD-L1 may restore T-cell function by blocking the interaction of PD-L1 with PD-1 and with CD80 on the surface of T cells. Furthermore, anti-PD-L1 sustains the PD-1/PD-L2 interaction, which may be important in maintaining immune homeostasis and potentially preventing autoimmunity. ${ }^{34}$ In a well-defined clinical study of 115 patients with CC, PD-L1 and PD-L2 were expressed in $19 \%$ and $29 \%$ of cervical tumors, respectively. Furthermore, PD- 1 was expressed by $>50 \%$ of tumor-infiltrating CD8+ T cells and CD4+FOXP3+ T cells, irrespective of PD-L1/L2 tumor expression. PD-L1 expression did not directly correlate with OS; however, subgroup analysis revealed improved survival in patients with a strong Treg response when the tumor was $\mathrm{PD}-\mathrm{Ll}+(P=.033){ }^{35}$

On the contrary, in a CC study from Leiden University, disease-free survival $(P=.022)$ and disease-specific survival $(P=.046)$ were significantly poorer in patients with squamous cell carcinoma and diffuse PD-L1 expression compared with marginal PD-L1 expression. ${ }^{36} \mathrm{PD}-\mathrm{L} 1+$ immune cells were in greater abundance surrounding metastatic lymph nodes, consistent with reports that advanced disease has higher levels of PD-L1 expression. ${ }^{36,37}$

lonizing Radiation, the Immune System, and the Abscopal Effect

Traditionally, ionizing radiation was believed to act as an immunosuppressant, exerting its cytotoxic antimitotic effects on tumor cells through DNA damage. ${ }^{38,39}$ However, in the 1950s, Mole ${ }^{40}$ described the immunomodulato- ry effects of radiation through the bystander or abscopal effect. A growing body of literature shows that radiation induces immunomodulatory effects, changing the tumor microenvironment and upregulating the inflammatory cascade, and is capable of boosting the abscopal effect. ${ }^{41}$ Several studies have shown an abscopal effect when RT is added to immunotherapy, likely due to RT-induced neoantigen release, which stimulates an antitumor immune response ${ }^{41} \mathrm{RT}$ increases TIL density by inducing chemokines, cytokines, and changes in tumor vasculature adhesion molecules, which facilitate T-cell homing and tumor infiltration. ${ }^{41-43}$ For example, tumor necrosis factor $\alpha($ TNF- $\alpha)$ was shown to have a synergistic effect when RT was combined with TNF- $\alpha$-based gene therapy in the human prostate cancer PC-3 xenograft, ${ }^{44}$ and can also inhibit tumor angiogenesis. ${ }^{45}$ However, the limited safety profile has slowed the clinical progression of TNF- $\alpha$ therapy. ${ }^{39,44,45}$

RT also affects dendritic cell migration and cross-presentation of tumor antigens, resulting in T-cell activation and proliferation ${ }^{43}$ and increased TCR diversity. ${ }^{46}$ Antigen release is enhanced by dendritic activation through the release of heat shock proteins, and accelerated dendritic maturation, migration, and phagocytosis via membrane-bound calreticulin signaling. ${ }^{39,47}$ Effector $\mathrm{T}$ cells are also recruited via chemokines such as CXCL- $16 .{ }^{48}$ The ability of antiangiogenic agents to normalize tumor vasculature, increase lymphocyte infiltration, limit hypoxia, improve dendritic cell maturation, reduce regulatory $\mathrm{T}$ cells, and transiently increase perfusion may produce an optimal tumor microenvironment for RT and immunotherapy to work synergistically against tumor cells, both locally and systemically. ${ }^{49}$

\section{Rationale for Multimodal Treatment: RT and}

\section{Immune Checkpoint Blockade}

Optimal results with immunotherapy may occur with multimodality treatment options, including dual checkpoint blockade or RT to overcome resistance mechanisms. Immunotherapy prior to RT may prime the immune system for antigen release and help reverse T-cell exhaustion. RT has been reported to increase PD-L1 expression, thus providing rationale for the use of agents targeting PD-1/PD-L1 as a combined modality strategy with RT. ${ }^{41,50,51}$ RT in combination with CTLA-4 blockade has been reported to induce responses in patients whose disease failed to respond to checkpoint blockade alone. ${ }^{52}$ In a seminal study of 22 patients with metastatic melanoma treated with combination anti-CTLA- 4 and RT, resistance was common, but tumor regression was seen in both the radiated index lesion and unirradiated lesions. ${ }^{46}$ Twyman-Saint Victor et $\mathrm{al}^{46}$ showed that patients with high PD-L1 expression showed persistent T-cell exhaustion with continued disease progression despite 
combined modality anti-CTLA-4 and RT. Results of this study suggest that a combination of various ICIs with RT may allow for increasing immune target diversity, thereby promoting an increased tumor response.

\section{Radiation and Immune Checkpoint}

Blockade Sequencing

Although data are limited regarding the optimal RT timing, dose, and fractionation schedule to evoke an ideal immune response when combined with immunotherapy, preclinical data are available. In a mouse model evaluating immune-mediated inhibition of metastasis, combined RT and anti-CTLA-4 provided a significant increase in survival compared with the other groups. ${ }^{53}$ In another preclinical study, combined RT and anti-PDL1 promoted tumor immunogenicity in mice and decreased the growth of secondary tumors. ${ }^{51}$ Another study showed that combined flank RT and systemic FMS-like tyrosine kinase receptor 3 ligand (FLT3L) therapy led to significant growth delay of the irradiated lesion and an abscopal effect, which was dependent on the presence of T cells, suggesting a synergistic effect between RT and checkpoint blockade. ${ }^{54}$

The sequencing of immunotherapy with RT has also been preliminarily studied, showing that anti-CTLA-4 therapy was most effective when given prior to RT, likely due to depletion of Tregs. ${ }^{55}$ In this study, RT (20 Gy) was delivered as a single fraction to the tumor-bearing site alone and with anti-CTLA-4 administered as a single intraperitoneal dose. The optimal sequencing of immunotherapy and RT in patients with CC, and the optimal RT fraction size and dose schedule, remain unknown.

\section{Clinical Trials of Immunotherapy}

\section{Therapy Targeting Anti-CTLA-4}

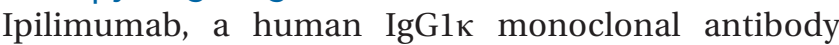
specific for CTLA-4, blocks CTLA-4 on the surface of activated $\mathrm{T}$ cells and Tregs. In a phase I/II study investigating ipilimumab in patients with recurrent and metastatic CC, a phase I safety lead-in was performed in 6 patients at $3 \mathrm{mg} / \mathrm{kg}$ for 4 cycles given every 21 days. ${ }^{56}$ Thereafter, 32 patients were treated with $10 \mathrm{mg} / \mathrm{kg}$ ipilimumab every 21 days for 4 cycles, followed by 4 cycles of maintenance therapy every 12 weeks, with a primary end point of radiographic response.$^{56}$ Toxicities were manageable and consisted of grade $\geq 3$ diarrhea $(n=4)$ and colitis $(n=1) .{ }^{56}$ Of the 34 evaluable patients, 1 had a partial response (PR), 10 had stable disease, and $23 \mathrm{had}$ progressive disease. Median PFS was 2.5 months $(95 \%$ CI, 2.1-3.2 months) and median OS was 8.5 months (95\% CI, 3.6 months to not reached). ${ }^{56}$ Although the activity of single-agent ipilimumab appears to be limited, the rationale remains for exploring combined strategies with this agent in an effort to achieve improved disease response and tumor control.

More recently in the phase I GOG 9929 trial, investigators examined the safety and tolerability of CRT and ipilimumab in patients with locally advanced, node-positive CC. Patients received standard CRT followed by 4 sequential ipilimumab cycles every 3 weeks. Primary end points included safety, tolerability, and identification of the maximum tolerated ipilimumab dose after extended-field RT. Secondary end points included PFS, patterns of failure, and translational studies, including PD-1 expression, and HPV-subtype-specific T-cell enumeration and characterization. Preliminary safety and tolerability results presented at the 2017 ASCO Annual Meeting showed that the maximum tolerated dose was $10 \mathrm{mg} / \mathrm{kg}$ and had manageable toxicity, with acute grade 3 toxicity in $10 \%$ of patients (2/19), which was self-limited. ${ }^{57}$ Based on Kaplan-Meier estimates, 12 -month OS and PFS rates were $90 \%$ and $81 \%$, respectively. Furthermore, CRT and sequential ipilimumab therapy increased PD- 1 expression. ${ }^{57}$

\section{Anti-PD-1 and Anti-PD-L1 Monotherapy}

Pharmacologic inhibitors of PD-1 have demonstrated antitumor activity and are currently under clinical exploration in multiple disease sites (see supplemental eTable $\underline{1}$, available with this article at JNCCN.org).$^{58}$

In June 2018, the FDA granted approval for the use of pembrolizumab in PD-L1+ CC based on the results of KEYNOTE-158 (ClinicalTrials.gov identifier: NCT02054806), which evaluated pembrolizumab in patients with recurrent or metastatic CC, ${ }^{59}$ and KEYNOTE-028 (NCT02054806), which evaluated patients with advanced PD-L1+ solid tumors (including CC). ${ }^{60}$ In KEYNOTE-158, 98 patients with an ECOG performance status of 0 to 1 were treated with 200 $\mathrm{mg}$ of pembrolizumab intravenously every 3 weeks for up to 24 months or until confirmed disease progression, intolerable toxicity, or death. PD-L1-positivity, defined by PDL1 expression score $\geq 1$, was seen in $83 \%$ of patients. Treatment-related toxicity was generally acceptable, with $11 \%$ of patients having grade $3 / 4$ adverse events (AEs) ${ }^{59}$ At a median follow-up of 10.3 months, the overall response rate (ORR) was $13 \%$, with complete response seen in 3 patients and PR in 10 patients; 17 patients had stable disease and the disease control rate was $31 \%$. Of those who experienced response, nearly $70 \%(9 / 13)$ had a response lasting $>9$ months. ${ }^{59}$ Responses were only seen in patients with PD-L1+ tumors, leading to FDA approval of pembrolizumab in this patient population. PD-L1 status on the study was determined using the IHC 22C3 pharmDx kit (Agilent Technologies, Inc.), which was approved as a companion diagnostic. Despite the limited response rate, the duration of response in a cohort of heavily pretreated patients remains encouraging.

Additionally, in the CC cohort of KEYNOTE-028, 24 patients with advanced PD-L1+ tumors $(\geq 1 \%$ expression 
IHC 22C3 antibody) received pembrolizumab, $10 \mathrm{mg} / \mathrm{kg}$ intravenously every 2 weeks for up to 24 months. ${ }^{60} \mathrm{Re}-$ sponse rates were assessed every 2 months until 6 months, and every 3 months thereafter; ORR was the primary end point. At the data cutoff, with a median follow-up of 11 months, the ORR was $17 \%$ in this heavily pretreated cohort, with $17 \%(4 / 24)$ experiencing a PR and $13 \%(3 / 24)$ with stable disease; 5 patients (21\%) had grade 3 AEs, but no patient had grade $4 / 5$ toxicity.

Taken together, pembrolizumab has shown single-agent activity and promising clinical outcomes with acceptable toxicity in patients with an overall poor prognosis and limited therapeutic options. Currently accruing clinical trials seek to further improve on these results and combine anti-PD-1 with traditional systemic agents and RT for further clinical benefit and safety, as shown in supplemental eTable 2.

Results of the phase I/II CheckMate 358 study of nivolumab in CC (ClinicalTrials.gov identifier: NCT02488759) were presented at the 2017 ASCO Annual Meeting. ${ }^{61}$ Patients with recurrent or metastatic CC with $\leq 2$ prior systemic therapies were treated with nivolumab, $240 \mathrm{mg}$ intravenously every 2 weeks until progression or unacceptable toxicity. ${ }^{61}$ Among the 19 patients with CC, ORR was $26.3 \%$, regardless of PDL1 expression, with a disease control rate of $68 \%$, suggesting encouraging clinical activity and a manageable safety profile. ${ }^{61}$

\section{Anti-PD-1 and Anti-PD-L1 Combinations}

Given the limited activity of single agents targeting PD-1 or PD-L1 in CC, several trials are exploring various combinations both as first-line therapy and in the recurrent/metastatic setting. One phase II study is exploring atezolizumab in combination with bevacizumab in patients with recurrent, persistent, or metastatic CC (ClinicalTrials.gov identifier: NCT02921269), with a primary end point of ORR. Another randomized phase II study comparing doxorubicin alone, atezolizumab alone, and doxorubicin + atezolizumab in recurrent CC (NCT03340376) has a primary end point of PFS and secondary end point of OS. The currently active GOG 3016 trial (NCT03257267) is a randomized phase III trial of anti-PD-1 (REGN2810) versus investigator's choice chemotherapy in patients with recurrent or metastatic CC, with the primary objective to compare OS between arms.

Additionally, several studies are exploring PD-1 and PD-L1 inhibitors as first-line therapy. The phase I PAPAYA trial is evaluating concurrent pembrolizumab with RT and cisplatin in newly diagnosed stage IBIVA CC (NCT03144466). Similarly, the NRG Oncology Group is opening GY017, a phase I study of atezolizumab in combination with CRT in patients with newly diagnosed, locally advanced CC. The study will evaluate 2 different schedules of atezolizumab dosing in relation to CRT, and seeks to determine whether administration of atezolizumab before RT enhances tumor control and antitumor immunity, using TCR clonality as a primary end point.

\section{Recombinant Listeria monocytogenes Vaccine}

Listeria monocytogenes is a $\beta$-hemolytic, gram-positive, facultative intracellular bacterium that has been used to study cell-mediated immunity. ${ }^{62}$ Listeria infects APCs and replicates within the cytoplasm of the host through degradation of the phagosomal membrane. Basu et $\mathrm{al}^{63}$ recently published the findings from their phase II trial of ADXS11-001 \pm cisplatin in the treatment of 109 patients with CC who previously received chemotherapy, RT, or CRT. Patients were randomized to either 3 or 4 doses of ADXS11-001 with intravenous cisplatin chemotherapy $\left(40 \mathrm{mg} / \mathrm{m}^{2}\right)$. Intergroup median OS, PFS, and ORR were comparable; however, 12 - and 18 -month OS rates were $30.9 \%$ versus $38.9 \%$ and $23.6 \%$ versus $25.9 \%$, respectively. AEs were mild-to-moderate and unrelated to treatment. ${ }^{63}$ In the similar phase II GOG 0265 study, ${ }^{64} 50$ patients with recurrent or metastatic CC received 3 doses of ADXS11001 , with a reported 12-month OS of $38 \%$. Although the activity of ADXS11-001 as a single agent appears to be limited, combinations with other agents, including chemotherapy and ICIs, may be warranted.

\section{Adoptive T-Cell Therapy}

Adoptive T-cell therapy has been explored in CC. In a study by Stevanovíc et al, ${ }^{12}$ patients with metastatic HPV-positive cancers were infused with TILs selected for HPV E6 and E7 reactivity (HPV-TILs). Of the 9 patients in the study, 3 experienced response (1 PR and 2 complete responses), with complete responses lasting at least 15 and 22 months at the time of publication. ${ }^{12}$ A phase II study evaluating engineered TCR targeting HPV E7 is ongoing (ClinicalTrials.gov identifier: NCT02858310).

\section{Conclusions}

There is an unmet clinical need to identify effective strategies for patients with locally advanced CC that does not respond to treatment with standard CRT approaches. Immunotherapy is a promising option for these patients given that most CCs are caused by high-risk HPV encoding 2 defined tumor-specific viral antigens, E6 and E7, which are constitutively expressed in each cancer cell. A number of immunotherapeutic options exist for treatment of CC, including checkpoint blockade with anti-CTLA-4, anti-PD-1, anti-PDL-1, recombinant Listeria monocytogenes vac- 
cine, and adoptive T-cell therapy. Further studies are needed to refine understanding of the immune system within CC, and to inform the optimal timing of immunotherapy and RT.

Developmental therapeutic options include combining ICIs or costimulatory activators to improve antitumor activity, use of new ICIs, identification of novel immunomodulatory targets, and implementation of validated biomarkers to leverage currently available therapeutic agents. Results of clinical trials to date have demonstrated that immunotherapy shows promise, and the studies reviewed herein demonstrate an opportunity for outcome improvement in locally advanced and metastatic CC.

\section{Acknowledgments}

The authors wish to acknowledge Dr. Arta Monjazeb for his contribution in generating Figure 1.

Submitted August 21, 2018; accepted for publication November 19, 2018.

Disclosures: Dr. Zamarin has disclosed that he served as the Co-Chair of the Cervical Cancer Translational Research Team at NRG Oncology, and is a consultant for and has a patent license with Merck. Dr. Mayadev has disclosed that she serves as the Co-Chair of the Cervix/Vulva Cancer Subcommittee at NRG Oncology, is a consultant for AstraZeneca, and serves on the GOG Foundation Advisory Board for ad-hoc clinical trial meetings. The remaining authors have disclosed that they have no financial interests, arrangements, affiliations, or commercial interests with the manufacturers of any products discussed in this article or their competitors.

Correspondence: Jyoti M. Mayadev, MD, Department of Radiation Medicine, University of California San Diego, 3855 Health Sciences Drive, La Jolla, CA 92093. Email: jmayadev@ucdavis.edu

\section{References}

1. American Cancer Society. Cancer Facts \& Figures 2015. Atlanta, GA American Cancer Society; 2015

2. Hreshchyshyn MM, Aron BS, Boronow RC, et al. Hydroxyurea or placebo combined with radiation to treat stages IIIB and IV cervical cancer confined to the pelvis. Int J Radiat Oncol Biol Phys 1979;5:317-322.

3. Keys HM, Bundy BN, Stehman FB, et al. Cisplatin, radiation, and adjuvant hysterectomy compared with radiation and adjuvant hysterectomy for bulky stage IB cervical carcinoma. N Engl J Med 1999;340:1154-1161.

4. Morris M, Eifel PJ, Lu J, et al. Pelvic radiation with concurrent chemotherapy compared with pelvic and para-aortic radiation for high-risk cervical cancer. N Engl J Med 1999;340:1137-1143.

5. Rose PG, Bundy BN, Watkins EB, et al. Concurrent cisplatin-based radiotherapy and chemotherapy for locally advanced cervical cancer. N Engl J Med 1999;340:1144-1153

6. Stehman FB, Ali S, Keys HM, et al. Radiation therapy with or without weekly cisplatin for bulky stage 1B cervical carcinoma: follow-up of a Gynecologic Oncology Group trial. Am J Obstet Gynecol 2007;197:503. e1-6.

7. Vale CL, Tierney JF, Davidson SE, et al. Substantial improvement in UK cervical cancer survival with chemoradiotherapy: results of a Royal College of Radiologists' audit. Clin Oncol (R Coll Radiol) 2010;22:590-601.

8. Varia MA, Bundy BN, Deppe G, et al. Cervical carcinoma metastatic to para-aortic nodes: extended field radiation therapy with concomitant 5-fluorouracil and cisplatin chemotherapy: a Gynecologic Oncology Group study. Int J Radiat Oncol Biol Phys 1998;42:1015-1023.

9. Randall LM, Monk BJ, Darcy KM, et al. Markers of angiogenesis in highrisk, early-stage cervical cancer: a Gynecologic Oncology Group study. Gynecol Oncol 2009;112:583-589.

10. Tewari KS, Sill MW, Long HJ III, et al. Improved survival with bevacizumab in advanced cervical cancer. N Engl J Med 2014;70:734-743.

11. Chung HC, Schellens JH, Delord JP, et al. Pembrolizumab treatment of advanced cervical cancer: updated results from the phase $2 \mathrm{KEY}$ NOTE-158 study [abstract]. J Clin Oncol 2018;32(Suppl):Abstract 5522.

12. Stevanovic $\mathrm{S}$, Draper LM, Langhan MM, et al. Complete regression of metastatic cervical cancer after treatment with human papillomavirus-targeted tumor-infiltrating T cells. J Clin Oncol 2015;33:1543-1550.

13. Clifford GM, Smith JS, Plummer M, et al. Human papillomavirus types in invasive cervical cancer worldwide: a meta-analysis. $\mathrm{Br} \mathrm{J}$ Cancer 2003;88:63-73.

14. Munoz N, Bosch FX, de Sanjose S, et al. Epidemiologic classification of human papillomavirus types associated with cervical cancer. N Engl J Med 2003;348:518-527.

15. Cogliano V, Baan R, Straif $K$, et al. Carcinogenicity of human papillomaviruses. Lancet Oncol 2005;6:204.

16. Munger K, Howley PM. Human papillomavirus immortalization and transformation functions. Virus Res 2002;89:213-228.

17. Sanclemente G, Gill DK. Human papillomavirus molecular biology and pathogenesis. J Eur Acad Dermatol Venereol 2002;16:231-240.

18. Eskander RN, Tewari KS. Immunotherapy: an evolving paradigm in the treatment of advanced cervical cancer. Clin Ther 2015;37:20-38.
19. Carson S. Human papillomatous virus infection update: impact on women's health. Nurse Pract 1997;22:24-25, 28-30, 35-37.

20. Mueller N. Overview of the epidemiology of malignancy in immune deficiency. J Acquir Immune Defic Syndr 1999;21(Suppl 1):S5-10.

21. Su TH, Chang TY, Lee YJ, et al. CTLA-4 gene and susceptibility to human papillomavirus-16-associated cervical squamous cell carcinoma in Taiwanese women. Carcinogenesis 2007;28:1237-1240.

22. Greenfield EA, Nguyen KA, Kuchroo VK. CD28/B7 costimulation: a review. Crit Rev Immunol 1998;18:389-418

23. Kazemi T, Younesi V, Jadidi-Niaragh F, Yousefi M. Immunotherapeutic approaches for cancer therapy: an updated review. Artif Cells Nanomed Biotechnol 2016;44:769-779.

24. Romano E, Kusio-Kobialka M, Foukas PG, et al. Ipilimumab-dependent cell-mediated cytotoxicity of regulatory $T$ cells ex vivo by nonclassical monocytes in melanoma patients. Proc Natl Acad Sci U S A 2015;112:6140-6145

25. Butte MJ, Keir ME, Phamduy TB, et al. Programmed death-1 ligand 1 interacts specifically with the B7-1 costimulatory molecule to inhibit $\mathrm{T}$ cell responses. Immunity 2007;27:111-122.

26. Tsushima F, Yao S, Shin T, et al. Interaction between B7-H1 and PD-1 determines initiation and reversal of T-cell anergy. Blood 2007;110:180-185.

27. Topalian SL, Drake CG, Pardoll DM. Targeting the PD-1/B7-H1(PD-L1) pathway to activate anti-tumor immunity. Curr Opin Immunol 2012;24:207-212.

28. Chen DS, Mellman I. Oncology meets immunology: the cancer-immunity cycle. Immunity 2013;39:1-10.

29. Ghebeh H, Mohammed S, Al-Omair A, et al. The B7-H1 (PD-L1) T lymphocyte-inhibitory molecule is expressed in breast cancer patients with infiltrating ductal carcinoma: correlation with important high-risk prognostic factors. Neoplasia 2006;8:190-198.

30. Hamanishi J, Mandai M, Iwasaki M, et al. Programmed cell death 1 ligand 1 and tumor-infiltrating CD8+ T lymphocytes are prognostic factors of human ovarian cancer. Proc Natl Acad Sci U S A 2007;104:3360-3365

31. Thompson RH, Kuntz SM, Leibovich BC, et al. Tumor B7-H1 is associated with poor prognosis in renal cell carcinoma patients with long-term follow-up. Cancer Res 2006;66:3381-3385.

32. Wu C, Zhu Y, Jiang J, et al. Immunohistochemical localization of programmed death-1 ligand-1 (PD-L1) in gastric carcinoma and its clinical significance. Acta Histochem 2006;108:19-24.

33. Ohigashi Y, Sho M, Yamada Y, et al. Clinical significance of programmed death-1 ligand-1 and programmed death-1 ligand-2 expression in human esophageal cancer. Clin Cancer Res 2005;11:2947-2953.

34. Akbari O, Stock P, Singh AK, et al. PD-L1 and PD-L2 modulate airway inflammation and iNKT-cell-dependent airway hyperreactivity in opposing directions. Mucosal Immunol 2010:3:81-91.

35. Karim R, Jordanova ES, Piersma SJ, et al. Tumor-expressed B7-H1 and $B 7-D C$ in relation to PD-1+ T-cell infiltration and survival of patients with cervical carcinoma. Clin Cancer Res 2009:15:6341-6347.

36. Heeren AM, Punt S, Bleeker MC, et al. Prognostic effect of different PD-L1 expression patterns in squamous cell carcinoma and adenocarcinoma of the cervix. Mod Pathol 2016;29:753-763.

37. Chen Z, Pang N, Du R, et al. Elevated expression of programmed death-1 and programmed death ligand-1 negatively regulates 
immune response against cervical cancer cells. Mediators Inflamm 2016;2016:6891482.

38. Demaria S, Bhardwaj N, McBride WH, et al. Combining radiotherapy and immunotherapy: a revived partnership. Int J Radiat Oncol Biol Phys 2005;63:655-666.

39. Levy A, Chargari C, Cheminant M, et al. Radiation therapy and immunotherapy: implications for a combined cancer treatment. Crit Rev Oncol Hematol 2013;85:278-287.

40. Mole RH. Whole body irradiation_radiobiology or medicine? Br J Radiol 1953;26:234-241.

41. Ngwa W, Irabor OC, Schoenfeld JD, et al. Using immunotherapy to boost the abscopal effect. Nat Rev Cancer 2018;18:313-322.

42. Hallahan D, Kuchibhotla J, Wyble C. Cell adhesion molecules mediate radiation-induced leukocyte adhesion to the vascular endothelium. Cancer Res 1996;56:5150-5155.

43. Sharabi AB, Lim M, DeWeese TL, et al. Radiation and checkpoint blockade immunotherapy: radiosensitisation and potential mechanisms of synergy. Lancet Oncol 2015;16:e498-509.

44. Chung TD, Mauceri HJ, Hallahan DE, et al. Tumor necrosis factor-alpha-based gene therapy enhances radiation cytotoxicity in human prostate cancer. Cancer Gene Ther 1998;5:344-349.

45. Mauceri HJ, Hanna NN, Wayne JD, et al. Tumor necrosis factor alpha (TNF-alpha) gene therapy targeted by ionizing radiation selectively damages tumor vasculature. Cancer Res 1996;56:4311-4314.

46. Twyman-Saint Victor C, Rech AJ, Maity A, et al. Radiation and dual checkpoint blockade activate non-redundant immune mechanisms in cancer. Nature 2015;520:373-377.

47. Hatfield P, Merrick A, Harrington K, et al. Radiation-induced cell death and dendritic cells: potential for cancer immunotherapy? Clin Oncol (R Coll Radiol) 2005;17:1-11.

48. Boon T, Coulie PG, Van den Eynde BJ, et al. Human T cell responses against melanoma. Annu Rev Immunol 2006;24:175-208.

49. Wong P, Houghton P, Kirsch DG, et al. Combining targeted agents with modern radiotherapy in soft tissue sarcomas. J Natl Cancer Inst 2014;106:dju329.

50. Dovedi SJ, Adlard AL, Lipowska-Bhalla G, et al. Acquired resistance to fractionated radiotherapy can be overcome by concurrent PD-L1 blockade. Cancer Res 2014;74:5458-5468.

51. Deng L, Liang H, Burnette B, et al. Irradiation and anti-PD-L1 treatment synergistically promote antitumor immunity in mice. J Clin Invest 2014;124:687-695.

52. Postow MA, Callahan MK, Barker CA, et al. Immunologic correlates of the abscopal effect in a patient with melanoma. N Engl J Med 2012;366:925-931.

53. Demaria S, Kawashima N, Yang AM, et al. Immune-mediated inhibition of metastases after treatment with local radiation and CTLA-4 blockade in a mouse model of breast cancer. Clin Cancer Res 2005;11:728-734.

54. Demaria $\mathrm{S}, \mathrm{Ng} \mathrm{B}$, Devitt $\mathrm{ML}$, et al. lonizing radiation inhibition of distant untreated tumors (abscopal effect) is immune mediated. Int J Radiat Oncol Biol Phys 2004;58:862-870.

55. Young $\mathrm{KH}$, Baird JR, Savage T, et al. Optimizing timing of immunotherapy improves control of tumors by hypofractionated radiation therapy. PLoS One 2016;11:e0157164.

56. Lheureux S, Butler MO, Clarke B, et al. Association of ipilimumab with safety and antitumor activity in women with metastatic or recurrent human papillomavirus-related cervical carcinoma. JAMA Oncol 2018;4:e173776.

57. Mayadev J, Brady WE, Lin YG, et al. A phase I study of sequential ipilimumab in the definitive treatment of node positive cervical cancer: GOG 9929 [abstract]. J Clin Oncol 2017;35(Suppl):Abstract 5526.

58. Adachi K, Tamada K. Immune checkpoint blockade opens an avenue of cancer immunotherapy with a potent clinical efficacy. Cancer Sci 2015;106:945-950.

59. Chung HC, Schellens JH, Delord JP, et al. Pembrolizumab treatment of advanced cervical cancer: updated results from the phase 2 KEYNOTE-158 study [abstract]. J Clin Oncol 2018;36(Suppl):Abstract 5522.

60. Frenel JS, Le Tourneau C, O'Neil B, et al. Safety and efficacy of pembrolizumab in advanced, programmed death ligand 1-positive cervical cancer: results from the phase lb KEYNOTE-028 trial. J Clin Oncol 2017;35:4035-4041.

61. Hollebecque A MT, Moore K et al. An open-label, multicohort, phase I/II study of nivolumab in patients with virus-associated tumors (CheckMate 358): efficacy and safety in recurrent or metastatic (R/M) cervical, vaginal, and vulvar cancers. J Clin Oncol 2017;35(Supp):Abstract 5504.

62. Wallecha A, Carroll KD, Maciag PC, et al. Multiple effector mechanisms induced by recombinant Listeria monocytogenes anticancer immunotherapeutics. Adv Appl Microbiol 2009;66:1-27.

63. Basu P, Mehta A, Jain M, et al. A randomized phase 2 study of ADXS11001 Listeria monocytogenes-listeriolysin $\mathrm{O}$ immunotherapy with or without cisplatin in treatment of advanced cervical cancer. Int J Gynecol Cancer 2018;28:764-772.

64. Huh WK, Dizon DS, Powell MA, et al. ADXS11-001 immunotherapy in squamous or non-squamous persistent/recurrent metastatic cervical cancer: results from stage I of the phase II GOG/NRG0265 study [abstract]. J Clin Oncol 2016;34(15 Suppl):Abstract 5516. 